
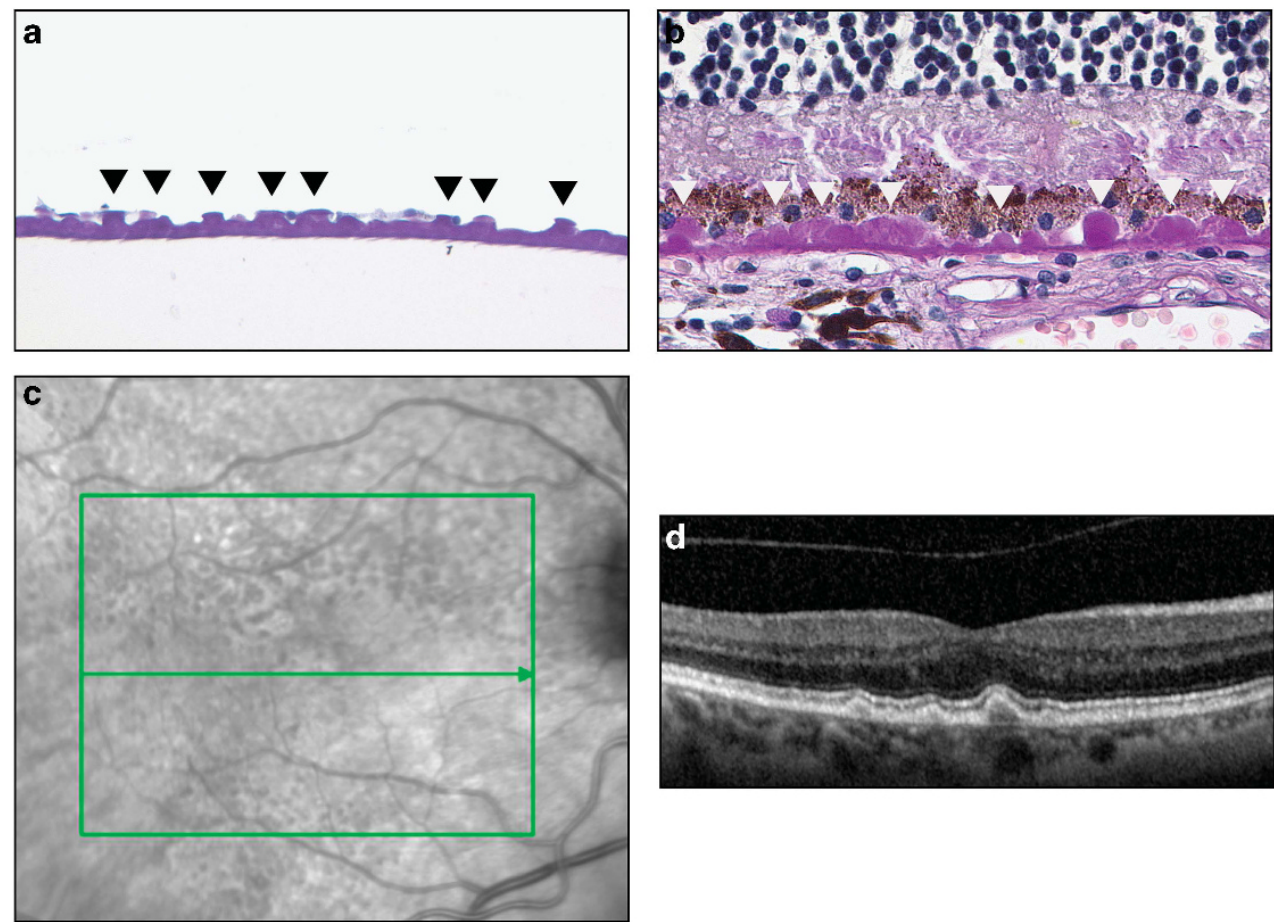

e Drusen (no / yes)

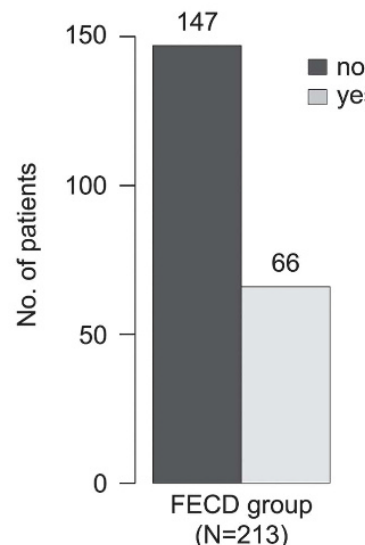

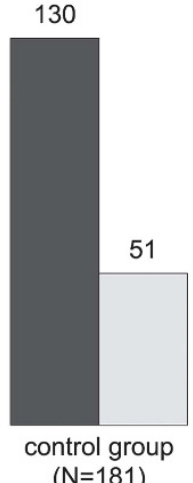

$\mathbf{f}$

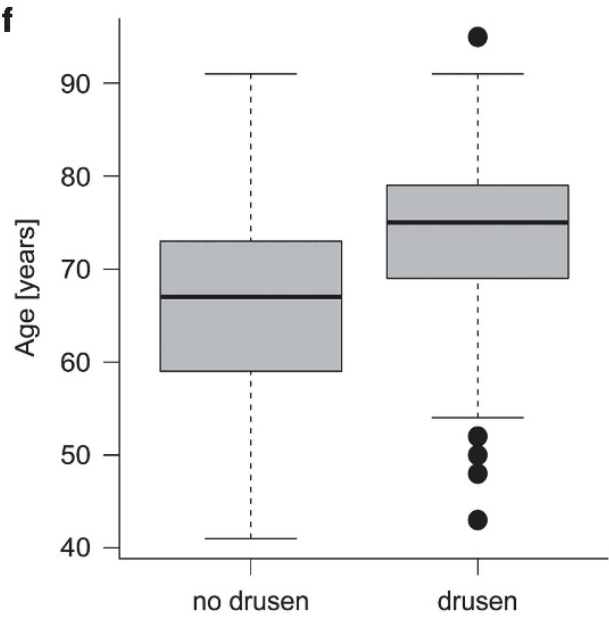

Figure 1 (a, b) Periodic acid-Schiff reaction staining of guttae (black triangle) of a stripped Descemet's membrane in Fuchs endothelial corneal dystrophy (FECD) and drusen (white triangle) in age-related macular degeneration (AMD). (c, d) Near-infrared reflectance (NIR, c) image and simultaneous spectral domain-optical coherence tomography (SD-OCT, d) of an eye with macular drusen. (e, f) Analysis of macular drusen in FECD and control group showed no significant difference between both groups (e). Presence of macular drusen correlated to patient age with higher presence of drusen in older patients (f).

Table 1 Patient demographics

\begin{tabular}{llll}
\hline & FECD & $\begin{array}{l}\text { Normal cornea } \\
\text { control }\end{array}$ & P-value \\
\hline$n_{\text {patients }}$ & 213 & 181 & - \\
Age \pm SD & $68.03 \pm 9.97$ & $68.21 \pm 11.76$ & $P=0.870$ \\
$\begin{array}{l}\text { Gender (female:male) } \\
n_{\text {eyes }}\end{array}$ & $116: 97$ & $96: 85$ & $P=0.857$ \\
$\begin{array}{l}\text { Phakiceyes: } \\
\text { pseudophakic }\end{array}$ & $262: 134$ & $263: 61$ & - \\
\hline
\end{tabular}

Eye (2018) 32, 840-841; doi:10.1038/eye.2017.301; published online 19 January 2018

Sir,

East of England regional retinopathy of prematurity service: lessons from the first year

Retinopathy of prematurity (ROP) affects $50 \%$ of babies screened and, of these, $4 \%$ will develop sight threatening 
(Type 1) ROP and require treatment. ${ }^{1}$ Recognition of progression to Type $1 \mathrm{ROP}$ and timely laser treatment is essential to prevent permanent visual loss, which accounts for $3 \%$ of childhood visual loss in the UK. ${ }^{2}$

Current issues with ophthalmologist delivered screening in the UK include recruitment difficulties, inexperience of screeners and the lack of a certified programme of continued medical education. Wide-field digital retinal imaging is an effective and accurate method for ROP telemedicine but the two commercially available cameras are expensive, making largescale NHS purchase unfeasible. ${ }^{3,4}$ An alternative is digital indirect ophthalmoscopy (DIO) which has been found to have between $94-100 \%$ sensitivity and $89-94 \%$ specificity for grading pre-plus or plus disease. ${ }^{5}$

The East of England neonatal network comprises 11 units referring to one treating centre, around one half of babies treated have been transferred from regional units specifically for ROP management (Figure 1). In 2016, nine of these units agreed to participate in an ROP telemedicine service. Implementation of the service reduced the number of unnecessary transfers from 56 to $21 \%$ and the proportion of treatments performed on babies with Type 2 disease from 21 to $7 \%$ (Figure 2). Three babies were transferred without images (two of these did not require treatment) and of those sent with images 17 out of 18 were gradable. Previously undiagnosed bilateral Stage 4 ROP was identified in one baby, enabling urgent transfer for vitreo-retinal surgery.

Problems have included inadequate, and sometimes obstructive, information technology (IT) support from participating hospitals which has delayed implementation in several units, and issues relating to image transfer, which is currently onerous for the referring ophthalmologist.

The DIO telemedicine service has saved $£ 21,000$ $(€ 23,300, \$ 27,500)$ in transport costs in the first year alone. The recent British Ophthalmic Surveillance Unit study indicated that nearly $30 \%$ of babies treated have Type 2 disease. ${ }^{1}$ Potentially unnecessary laser treatment for Type 2 ROP, with all the risks it entails, was prevented in most cases by telemedicine in this study.

Telemedicine for ROP using wide-angle digital retinal imaging is likely to remain the gold standard and enables screening by technicians and nurses. The advantages of the DIO based telemedicine service is its affordability: for the cost of one wide-angle retinal camera, seven systems were purchased. The disadvantage of DIO based telemedicine is the continued need for an ophthalmologist to screen.

This service describes one method for ROP telemedicine, but all methods require local and national IT support and the use of a digital network such as

Picture Archiving and Communications System (PACS). This would make telemedicine easier to use and facilitate image sharing and cross-cover between units and regions. Current difficulty with the recruitment and retention of experienced ophthalmologists underlines the importance of developing a telemedicine strategy to ensure optimal management and prevention of childhood blindness due to ROP.

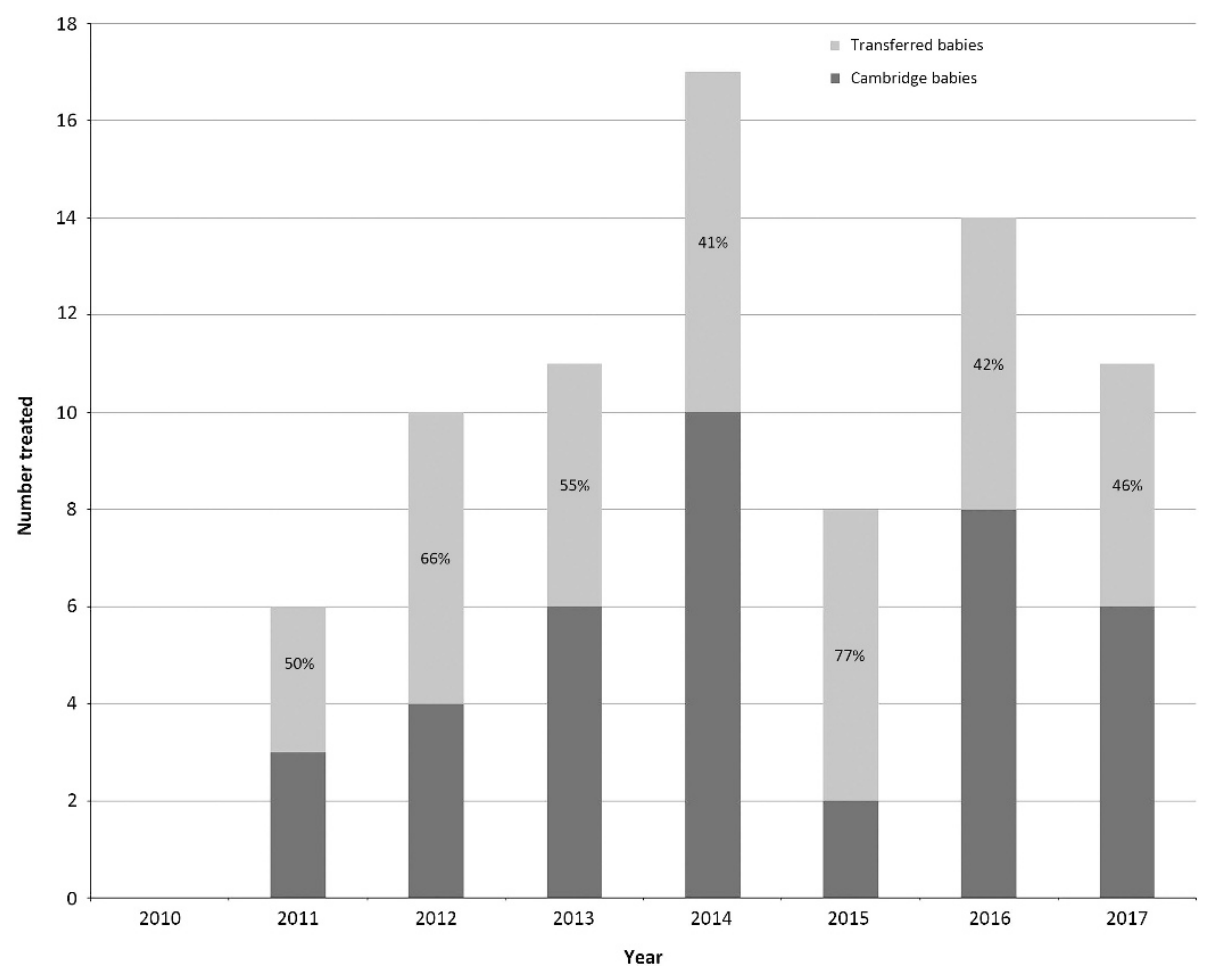

Figure 1 Proportion of babies treated for ROP who were transferred from regional units. 
a

Outcome From Transfer,- pre telemedicine $\mathrm{N}=43$ (2011-2015)

- Type 1 treated $\quad$ wasted transfer $=$ treated early

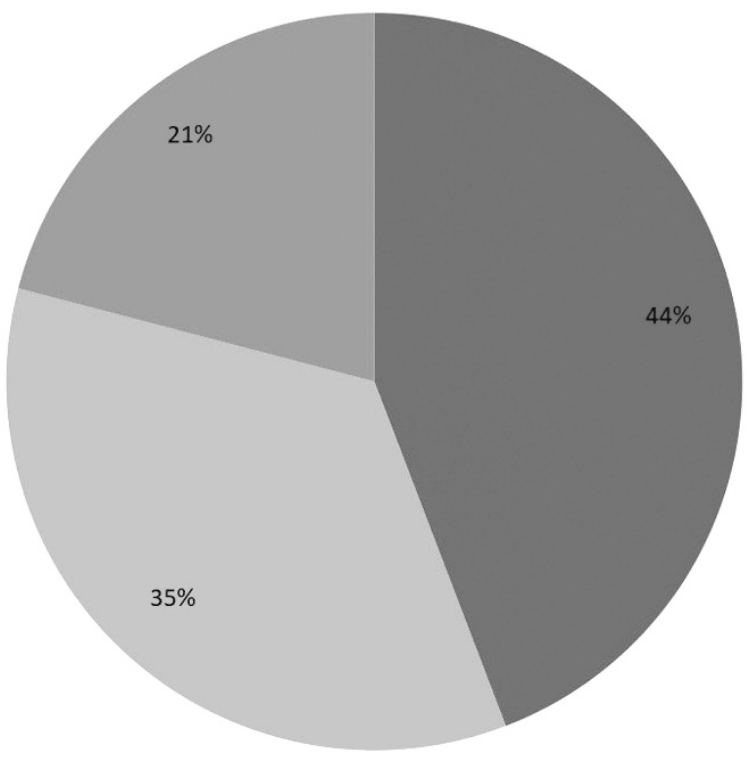

b

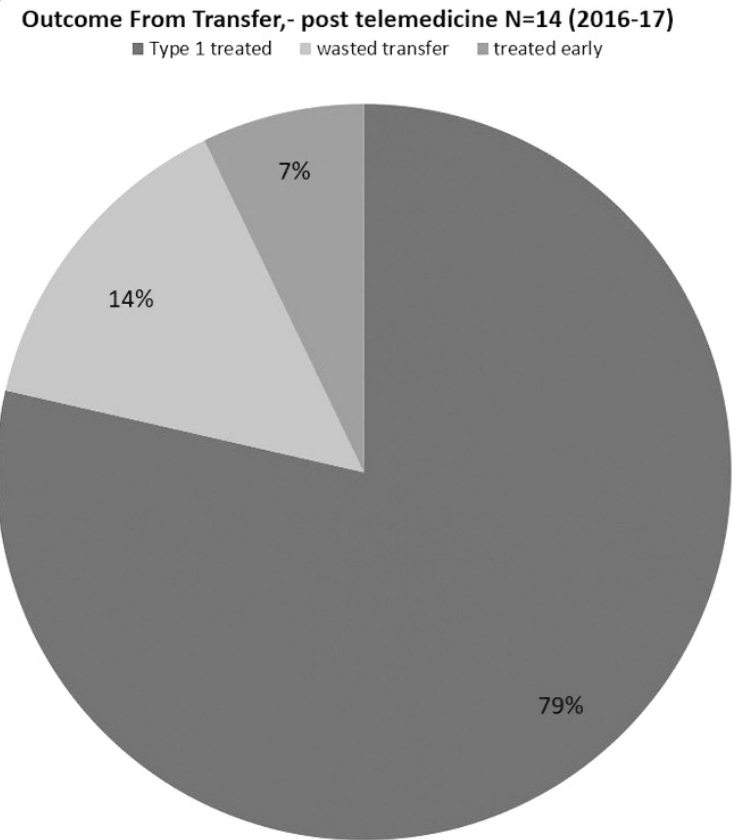

Figure 2 Pie charts showing outcome of transfer (a) pre and (b) post telemedicine service implementation.

\section{Conflict of interest}

The authors declare no conflict of interest.

\section{Acknowledgements}

We thank the donors to Addenbrooke's Charitable Trust for their generosity and the regional screeners for supporting the service.

\section{References}

1 Adams GGW, Bunce C, Xing W, Butler L, Long V, Reddy A et al. Treatment trends for retinopathy of prematurity in the UK: active surveillance study of infants at risk. BMJ Open 2017; 7: e013366.

2 Rahi JS, Cable N. Severe visual impairment and blindness in children in the UK. Lancet 2003; 362(9393): 1359-1365.

3 Fierson WM, Capone A. Telemedicine for evaluation of retinopathy of prematurity. Pediatrics 2015; 135(1): 238-253.

4 Wang SK, Callaway NF, Wallenstein MB, Henderson MT, Leng T, Moshfeghi DM. Sundrop: six years of screening for retinopathy of prematurity with telemedicine. CJO 2015; 50(2): 101-106.

5 Prakalapakorn SG, Wallace DK, Dolland RS, Freedman SF. Evaluation of the accuracy of grading indirect ophthalmoscopy video images for retinopathy of prematurity screening. J Paediatr Ophthalmol Strabismus 2015; 5292: 85-92.

LE Allen ${ }^{1}$ and A te Water Naudé ${ }^{2}$

${ }^{1}$ Ophthalmology Department, Cambridge University Hospital NHS Trust, Cambridge, UK
${ }^{2}$ University of Cambridge Medical School, Cambridge, UK

E-mail: Louise.allen@addenbrookes.nhs.uk

Purchase of telemedicine systems was made possible by donations to Addenbrooke's Charitable Trust.

Eye (2018) 32, 841-843; doi:10.1038/eye.2017.302; published online 19 January 2018

Sir,

A novel mutation (LEU396ARG) in OPA1 is associated with a severe phenotype in a large dominant optic atrophy pedigree

Dominant optic atrophy is a degenerative disease of the optic nerve, which causes decreased visual acuity in early childhood. Clinical signs include cecocentral scotomas, color blindness (blue-yellow dyschromatopsia), and temporal pallor of the optic nerve head. Visual acuity deficits are typically observed in the first decade of life and progress slowly. Visual acuity is highly variable, ranging from $20 / 20$ to count fingers.

$O P A 1$ is a nuclear gene that encodes a GTPase with key roles in mitochondial metabolism. Mutations in the OPA1 gene cause the majority of dominant optic atrophy cases. ${ }^{1,2}$ We previously reported features of a sixgeneration dominant optic atrophy family with a particularly severe phenotype. By the end of their second decade of life, visual acuities of family members with dominant optic atrophy ranged from 20/40 to 20/80, and most family members in their seventh decade of life or older were legally blind. ${ }^{3}$ Here we report testing this 